\title{
Determination of the radii of the circles which touch three given circles.
}

\section{By Alex. Holar, M.A.}

Let the points $\mathrm{A}, \mathrm{B}, \mathrm{C}$ be the centres of three given circles, whose radii are $a, b, c$; and let $d, e, f$ be the distances $\mathrm{BC}, \mathrm{CA}, \mathrm{AB}$ between the centres (Fig. 41). It is required to find the radii of the circles which touch the circles $\mathrm{A}, \mathrm{B}, \mathrm{C}$.

1. Suppose that $O$ is the centre and $x$ the radius of a circle which touches the circles $A, B, C$ all externally at the points $P, Q, R$.

Then $\mathrm{OA}, \mathrm{OB}, \mathrm{OC}$ pass through the points of contact.

$$
\therefore \quad \mathrm{OA}=x+a, \mathrm{OB}=x+b, \mathrm{OC}=x+c .
$$

Let $\mathrm{QR}$, the straight line joining the points of contact of the tangent circle $O$ with the circles $B, C$, cut those circles again at $Q^{\prime}, R^{\prime}$, and meet $\mathrm{BC}$ in $\mathrm{S}$. Then $\mathrm{S}$ is the direct centre of similitude of the circles $\mathbf{B}, \mathbf{C}$.

$\therefore \quad \mathrm{CQ}^{\prime}$ is parallel to $\mathrm{BQ}$ and $\mathrm{BR}^{\prime}$ to $\mathrm{CR}$.

$$
\begin{gathered}
\therefore \frac{\mathrm{QR}}{\mathrm{QQ}^{\prime}}=\frac{\mathrm{OR}}{\mathrm{OC}}=\frac{x}{x+c} \text { and } \frac{\mathrm{QR}}{\mathbf{R}^{\prime} \mathrm{R}}=\frac{\mathrm{OQ}}{\mathrm{OB}}=\frac{x}{x+b} . \\
\therefore \quad \frac{\mathrm{QR}^{2}}{\mathrm{QQ}^{\prime} \cdot \mathbf{R}^{\prime} \mathbf{R}}=\frac{x^{2}}{(x+b)(x+c)} .
\end{gathered}
$$

Now $Q Q^{\prime} \cdot R^{\prime} R$ is constant for any secant through the centre of similitude $\mathrm{S}$.

Hence if $l$ is the length of the direct common tangent TT' passing through $\mathrm{S}$,

$$
\mathrm{QQ} \cdot \mathrm{R}^{\prime} \mathrm{R}=\mathrm{TT}^{\prime \prime 2}=l^{2}=d^{2}-(b-c)^{2} .
$$

Also if $m$ and $n$ are the lengths of the direct common tangents to circles $\mathbf{C}, \mathbf{A}$, and $\mathbf{A}, \mathbf{B}$,

$$
m^{2}=e^{2}-(c-a)^{2}, n^{2}=f^{2}-(a-b)^{2} .
$$

Then, denoting the sides of triangle $\mathrm{PQR}$ by $p, q, r$, we have

$$
p^{2}=\frac{l^{2} x^{2}}{(x+b)(x+c)}, \quad q^{2}=\frac{m^{2} x^{2}}{(x+c)(x+a)}, \quad r^{2}=\frac{n^{2} x^{2}}{(x+a)(x+b)},
$$


Now $x$ is the radius of the circumcircle of triangle PQR

$$
\begin{aligned}
\therefore \quad \frac{p q r}{4 \Delta_{p q r}} & =x . \\
\therefore \quad \frac{16 \Delta_{p q r}^{2}}{p^{2} q^{2} r_{1}^{2}} & =\frac{1}{x^{2}} . \\
\frac{2 q^{2} r^{2}+2 r^{2} p^{2}+2 p^{2} q^{2}-p^{4}-q^{4}-r}{p^{2} q^{2} r^{2}} & =\frac{1}{x^{2}} . \\
\frac{2}{p}+\frac{2}{q}+\frac{2}{r^{2}}-\frac{p^{2}}{q^{2} r^{2}}-\frac{q^{2}}{r^{2} p^{2}}-\frac{r^{2}}{p^{2} q^{2}} & =\frac{1}{x^{2}} .
\end{aligned}
$$

Substitute the above values of $p^{2}, q^{2}, r^{2}$.

$$
\begin{aligned}
& \therefore \quad \frac{2(x+b)(x+c)}{l^{2}}+\frac{2(x+c)(x+a)}{m^{2}}+\frac{2(x+a)(x+b)}{n^{2}} \\
& \quad-\frac{l^{2}}{m^{2} n^{2}}(x+a)^{2}-\frac{m^{2}}{n^{2} l^{2}}(x+b)^{2}-\frac{n^{2}}{l^{2} m^{2}}(x+c)^{2}=1 . \\
& \therefore \quad\left(2 m^{2} n^{2}+2 n^{2} l^{2}+2 l^{2} m^{2}-l^{4}-m^{4}-n^{4}\right) x^{2} \\
& +2\left\{(b+c) m^{2} n^{2}+(c+a) n^{2} l^{2}+(a+b) l^{2} m^{2}-a l^{4}-b m^{4}-c n^{4}\right\} x \\
& +\left(2 b c m^{2} \iota^{2}+2 c a n^{2} l^{2}+2 a b l^{2} m^{2}-a^{2} l^{4}-b^{2} m^{4}-c^{2} n^{4}-l^{2} m^{2} n^{2}\right)=0,
\end{aligned}
$$

which is a quadratic equation to find $x$.

2. Solving the quadratic, the expression under the square root is

$$
\begin{aligned}
& 4\left\{(b+c) m^{2} n^{2}+(c+a) n^{2} l^{2}+(a+b) l^{2} m^{2}-a l^{4}-b m^{4}-c n^{4}\right\}^{2} \\
& -4\left(2 m^{2} n^{2}+2 n^{2} l^{2}+2 l^{2} m^{2}-l^{4}-m^{4}-n^{4}\right) \\
& \quad\left(2 b c m^{2} n^{2}+2 c a n^{2} l^{2}+2 a b l^{2} m^{2}-a^{2} l^{4}-b^{2} m^{4}-c^{2} n^{4}-l^{2} m^{2} n^{2}\right) .
\end{aligned}
$$

This expression is of the tenth degree, and can be resolved into ten linear factors.

If we put $l^{2}$ or $m^{2}$ or $n^{2}=0$, the expression vanishes ;

$$
\therefore d^{2}-(b-c)^{2}, e^{2}-(c-a)^{2}, f^{2}-(a-b)^{2} \text { are factors. }
$$

Again, if $d$ were equal to $e+f$, the centres of the three given circles would be in a straight line, which would be an axis of symmetry for each circle (Figs. 42, 43). Hence the two circles, which touch the given circles all externally (or all internally), would have equal radii.

Thus the two roots of the quadratic would be equal. 
Therefore the expression under the square root must vanish when $d=e+f$, or when $d^{2}=(e+f)^{2}$, since only even powers of $d$ occur in it.

Therefore $d+e+f$ and $-d+e+f$ are factors.

Similarly $d-e+f$ and $d+e-f$ are factors.

Hence the expression

$$
\begin{aligned}
& \equiv \mathbf{A}(d+b-c)(d-b+c)(e+c-a)(e-c+a) \\
& \quad(f+a-b)(f-a+b)(d+e+f)(-d+e+f)(d-e+f)(d+e-f),
\end{aligned}
$$

where $\mathbf{A}$ is some constant coefficient.

Putting

$a=0, b=0, c=0, d=1, e=1, f=1$, and thus $l^{2}=1, m^{2}=1, n^{2}=1$, we have $\quad 12=3 \mathrm{~A}$, so that $\mathrm{A}=4$.

Now $(d+e+f)(-d+e+f)(d-e+f)(d+e-f)=16 \Delta_{\text {def }}^{a}$, where $\Delta_{d e f}$ is the area of the triangle whose sides are $d, e, f$.

Therefore the expression under the square root is $64 l^{2} m^{2} n^{2} \Delta_{\text {des }}^{2}$, which is a remarkable form for it.

3. Thus the roots of the quadratic equation are

$$
x=\frac{a l^{4}+b m^{4}+c n^{4}-(b+c) m^{2} n^{2}-(c+a) n^{2} l^{2}-(a+b) l^{2} m^{2} \pm 4 l m n \Delta_{\text {def }}}{2 m^{2} n^{2}+2 n^{2} l^{2}+2 l^{2} m^{2}-l^{4}-m^{4}-n^{4}},
$$

where $l^{2}=d^{2}-(b-c)^{2}, \quad m^{2}=e^{2}-(c-a)^{2}, \quad n^{2}=f^{2}-(a-b) .^{2}$

This gives the radius of a tangent circle exterior to all the given circles.

4. To deduce the radii of the other tangent circles.

(1) For a tangent circle enveloping all the given circles,

$$
\mathrm{OA}=x-a, \quad \mathrm{OB}=x-b, \quad \mathrm{OC}=x-c .
$$

Hence the radius $x$ could be obtained by changing the signs of $a, b, c$ in $\$ 3$.

Now those changes would leave $l, m, n$ unaltered, and as $\Delta_{\text {lef }}$ remains constant, $x$ would be changed in sign only.

Therefore according as the roots in $\$ 3$ are real and

(i) both positive, (ii) one positive, the other negative, (iii) both negative, there will be

(i) two exterior tangent circles,

(ii) one exterior, and one enveloping tangent circle,

(iii) two enveloping tangent circles. 
Thus when the roots are real, there is a pair of tangent circles each of which has the given circles all on the same side of it.

(2) For a tangent circle enveloping circle $A$, but exterior to circles B, C,

$$
\mathrm{OA}=x-a, \quad \mathrm{OB}=x+b, \quad \mathrm{OC}=x+c .
$$

Hence the radius $x$ can be obtained by changing the sign of $a$ in $\$ 3$.

Those new roots with their signs changed belong to a tangent circle exterior to circle $\mathbf{A}$, but enveloping circles $\mathbf{B}, \mathbf{C}$.

For here

$$
\mathrm{OA}=x+a, \quad \mathrm{OB}=x-b, \quad \mathrm{OC}=x-c,
$$

so that $a, b, c$ have now opposite signs to the preceding.

Therefore according as the new roots are real and

(i) both positive, (ii) one positive, the other negative, (iii) both negative, there will be

(i) two tangent circles en veloping circle $\mathrm{A}$, but exterior to circles $\mathrm{B}, \mathrm{C}$,

(ii) one tangent circle

and one tangent circle exterior to circle $\mathrm{A}$, but enveloping circles $\mathrm{B}, \mathrm{C}$, (iii) two tangent circles

Therefore when those new roots are real, there is a pair of tangent circles each of which has circle $\mathrm{A}$ on one side and circles $\mathrm{B}, \mathrm{C}$ on the opposite side of it.

(3) To obtain the radii of the pair of tangent circles which have circle $\mathrm{B}$ on one side, and circles $\mathrm{C}, \mathrm{A}$ on the opposite side, change the sign of $b$ in $\$ 3$.

(4) To find the radii of the pair of tangent circles having circle $\mathrm{C}$ on one side, and circles $\mathrm{A}, \mathrm{B}$ on the opposite side, make $c$ negative in $\$ 3$.

Thus in general there are four pairs of tangent circles.

\section{Historical Note.}

5. In Leybourn's Mathematical Questions from the Ladies' Diary, Vol. IV., pp. 270-275, there is a solution of the proposed problem by Binet, taken from the Journal de l'Ecole Polytechnique, 17 Cahier, $t$. In the relation connecting the lengths of the six straight lines 
joining four points in a plane, Binet puts $x+a, x+b, x+c$ for the lengths of three lines that are concurrent, and $d, e, f$ for the lengths of the other three lines; $a, b, c$ being the radii of the three given circles, $d, e, f$ the distances between their centres, and $x$ the radius of a circle touching the given circles all externally.

After a long reduction a quadratic equation in $x$ with very complex coefficients is obtained, and no attempt is made to solve the quadratic.

We learn from Pappus that the problem "To describe a circle to touch three given circles" was the chief proposition in a lost work of Apollonius of Perga on Tangencies (c. 210 B.C.).*

Franciscus Vieta solved this problem at the close of the 16th century, $\dagger$ and since then it has occupied the attention of many mathematicians. In 1814 Gergonne, in the Annales de Mathématiques, t. vi., p. 439, gave a solution based on the theory of Radical Axis, Similitude, Pole and Polar. Gergonne's construction can also be applied, when one or two of the given circles become points or straight lines, and it seems to be the most general geometrical construction yet published. + For an enumeration of the different positions of the three given circles relatively to one another, with the number of possible tangent circles in each case, see an article by R. F. Muirhead "On the Number and Nature of the Solutions of the Apollonian Contact Problem" (Proc. Edin. Math. Soc., Vol. XIV., pp. 135-147).

*Cf. Hutton'^ Ifath. Dict. pp. 129, 130, or Leslie's Geometry, 1811, pp. 434-437.

†For Vieta's solution see Leybourn's Math. Quest., Vol. IV., pp. 262-264.

†Cf. Chasles' Géométrie Supérieure, XII. éd., pp. 498-501.

On the orthoptic locus of the semi-cubical parabola.

By A. G. Burgess, M.A. 